\title{
LEVANTAMENTO DAS INTOXICAÇÕES POR PARAQUATNO ESTADO DO PARANÁ NOS ANOS DE 1998, 1999 E 2000
}

\section{SURVEY ON PARAQUAT INTOXICATION IN PARANÁ STATE IN THE YEARS 1998, 1999 AND 2000}

\author{
CAVAШ,B. M. ${ }^{3} ;$ GABRIEL, M. M. ${ }^{1} \&$ LOPES, M.1,2
}

${ }^{1}$ Docente da Universidade Federal Do Parana - BRASIL
${ }^{2}$ Instituto Médico Legal Do Paraná
${ }^{3}$ Bolsista da Universidade Federal do Paraná (Bolsa Permanência)

\section{RESUMO}

O paraquaté um herbicida de contato, não seletivo e pertencente ao grupo químico dos bipindílios. Ë um dos mais específicos agentes tóxicos pulmonares conhecidos e possui uma alta taxa de mortalidade em casos de intoxicações, por isso tem sido objeto de muitas investigações em vários países. Com a finalidade de verificar a extensão da utilização do paraquat no Estado do Paraná realizamos um levantamento do número de casos e as causas das intoxic ações ocomidas nos anos de 1998 (20casos), 1999 (17 casos) e 2000 (28 casos).

Para isso foram utilizados dados obtidos em órgãos públicos como a Secretaria de Estado da Agricultura e Abastecimento (SEAB-PR) e o Centro de Saúde Ambiental (órgão vincula do à Sec retaria DE Estado da Saúde).

Palavras chaves: paraquat, intoxicação, herbicida.

\begin{abstract}
Paraquat is a contact non-selective herbic ide that belongs to the bipinililic chemical group. It is one of the most specific toxic agent to the lungs known so far and is responsible for a high mortality rate in cases of intoxication. Because of that, paraquat has been the object of much scientific investigation in several countries. In order to know the extension of paraquat use in the Parana State, it was done a search on the number of cases and the causes of intoxication occured in the years 1998 (20 cases), 1999 (17 cases) and 2000 (28 cases). The data were obtained from public institutions, such as Secretaria de Estado da Agricultura e Abastec imento and the Centro de Saúde Ambiental.

Key Words: paraquat, intoxication, herbicide.
\end{abstract}

\section{INTRODUÇÃO}

O extermínio de envas daninhas, por meios químicos, foi realizado até a Segunda Guerra Mundial, pormeio da utilização de soluções à base de ácidose sa is inorgânicosta is como: ácido sulfúnico, clorato de sódio, tróxido de arsênico, arsenito de sódio, derivados de petróleo, sulfatos de ferro e cobre ou borato de sódio, os quais são difíc eis de manusear, de elevada toxicidade e não específic os. (MIDIO \& MARTINS, 1997; CASAREIT\& DOUШ'S, 1996)

Em consequência aos problemas relacionados com a segurança na aplicação, soma dosà necessida de de uma certa seletividade no controle dasplantas daninhas, estudos foram realizadose surgiram os herbicidasorgânicos. Estes, atualmente, estão representados por 32 grupos químicos, dentre eles tem-se o grupo Bipindílios onde o paraquat é o representante mais importante. (ALMEIDA, F.S; RODRIGUES, BN., 1985; ASTOLFI, E Et.ali 1982; EUENHORN, 1997).

O paraquat, foi sintetizado em 1882, mas suas propriedades pesticidas não foram descobertas até 1959. É um herbicida de contato, não seletivo, sendo um dos mais específic os agentes tóxicos pulmonares conhecidos e apresenta uma alta taxa de morta lida de em casos de intoxic ação, porisso, tem sido objeto de muitos estudos. (CASAREIT \& DOUШ'S, 1996; LARINE, 1997) Inúmeros países tem banido ou restringido severamente o seu uso devido os risc os à vida humana. (CASAREIT\& DOUШ'S, 1996). 
Segundo o Sindic ato Nacional da Indústria de Defensivos Agńc olas, o Brasil, apresenta um grande consumo de pesticidas. Pode-se evidenciaresse fato avaliando os gastos com pesticidas nos meses de janeiro a agosto de 2000 que atingiu a cifra de $\$ 1.134 .757,00$. Somente no mês de agosto, foram gastosem pestic idas $\$ 296.188$ deste total ma is de $57 \%$ em herbicidas $(\$ 168.877,00)$.

O objetivo deste trabalho foi verificar o perfil das intoxicações por paraquat no Paraná pormeio de um levantamento da incidência e dascausas das intoxicaçõesocomidas nos anos de 1998 a 2000.

\section{MATERIAL E MÉTODOS}

Foi realizado um levantamento de dados junto a Secretaria de Estado da Agricultura e Abastecimento (SEAB-PR) e do Centro de Saúde Ambiental (Secretaria de Estado da Saúde).

Os dados obtidos foram orga nizadosem tabelas de a cordo com as informações das instituições acima . As três primeiras tabelas são referentes as intoxicações ocomidas nos anos de 1998, 1999 e 2000, onde consta: a substância ativa do herbicida, causas das intoxicaçõese evolução doscasose a quarta tabela cita os Municípios do PRonde ocorreram as intoxicações nos anos de 1998 e 1999. (CAVAШ, BM. 2000)

\section{RESULTADOS E DISCUSSÃO}

Informações obtidas junto à Sec retaria de Estado da Agricultura e Abastec imento do Paraná (SEAB) permitiram estimar quais os herbic idas mais utilizados no Estado, de acordo com o controle do receituário emitido pelos Engenheiros Agrônomos. Tendo-se como herbicidas mais utilizados: glifosa to, 2,4-D, paraquat, imasaquin, atrazinas e simazinas.

Astabelas 1, 2 e 3 mostram as intoxicaçõesocomidas, no estado do Paraná, nos anos de 1998, 1999 e 2000, substância ativa do herbicida, causas de intoxicação, ocupação dos intoxic ados e evolução dos casos.

TABELA 1: INTOXICAÇÕES POR PARAQUATNO PARANÁ NO ANO DE 1998

\begin{tabular}{|c|c|c|c|}
\hline & & Número de casos & Percentual \\
\hline \multirow{5}{*}{$\begin{array}{c}\text { Substância } \\
\text { ativa }\end{array}$} & Paraquat & 17 & $85 \%$ \\
\hline & Paraquat + fomesafen & 1 & $5 \%$ \\
\hline & Paraquat + glifosato & 1 & $5 \%$ \\
\hline & Paraquat + glifosato + dodecilbenzenosulfonato de sódio & 1 & $5 \%$ \\
\hline & TOTAL & 20 & $100 \%$ \\
\hline Causas de & Acidental & 3 & $15 \%$ \\
\hline \multirow{2}{*}{ intoxic ação } & Profissional & 11 & $55 \%$ \\
\hline & Suicídio & 6 & $30 \%$ \\
\hline Ocupação dos & Agricultor & 17 & $85 \%$ \\
\hline \multirow[t]{3}{*}{ intoxicados } & Ignorada & 2 & $10 \%$ \\
\hline & Outra & 1 & $1 \%$ \\
\hline & Cura com seqüela & 1 & $5 \%$ \\
\hline Evolução dos & Cura sem seqüela & 9 & $45 \%$ \\
\hline \multirow[t]{3}{*}{ casos } & Ignorado & 4 & $20 \%$ \\
\hline & Óbito (todos por suicídio) & 4 & $20 \%$ \\
\hline & Sem informações & 2 & $10 \%$ \\
\hline
\end{tabular}

FONTE: PARANÁ. Secretaria de Estado da Saúde. SESA - PR Centro de Saúde Ambiental. 
TABELA 2: INTOXICAÇÕES POR PARAQUATNO PARANÁ NO ANO DE 1999

\begin{tabular}{|c|c|c|c|}
\hline & & Número de casos & Percentual \\
\hline \multirow{3}{*}{$\begin{array}{c}\text { Substância } \\
\text { ativa }\end{array}$} & Paraquat & 16 & $94,1 \%$ \\
\hline & Paraquat + diuron & 1 & $5,9 \%$ \\
\hline & TOTAL & 17 & $100 \%$ \\
\hline \multirow{4}{*}{$\begin{array}{l}\text { Causas de } \\
\text { intoxicação }\end{array}$} & Acidental & 2 & $11,8 \%$ \\
\hline & Indeterminada & 1 & $5,9 \%$ \\
\hline & Profissional & 8 & $47,1 \%$ \\
\hline & Suicídio & 6 & $35,3 \%$ \\
\hline \multirow{5}{*}{$\begin{array}{l}\text { Ocupação dos } \\
\text { intoxicados }\end{array}$} & Agricultor & 12 & $70,6 \%$ \\
\hline & Ignorada & 3 & $17,6 \%$ \\
\hline & Menor (acidental) & 1 & $5,9 \%$ \\
\hline & Outra & 1 & $5,9 \%$ \\
\hline & Cura com seqüela & 1 & $5,9 \%$ \\
\hline \multirow{3}{*}{$\begin{array}{l}\text { Evolução dos } \\
\text { casos }\end{array}$} & Cura sem seqüela & 12 & $70,6 \%$ \\
\hline & Ignorado & 1 & $5,9 \%$ \\
\hline & Óbito (todos por suicídio) & 3 & $17,6 \%$ \\
\hline
\end{tabular}

FONIE: PARANÁ. Secretaria de Estado da Saúde. SESA - PR Centro de Saúde Ambiental.

TABELA 3: INTOXICAÇÕESPOR PARAQUATNO PARANÁ NO ANO DE 2000.

\begin{tabular}{|c|c|c|c|}
\hline & & Número de casos & Percentual \\
\hline \multirow{4}{*}{$\begin{array}{c}\text { Substância } \\
\text { ativa }\end{array}$} & paraquat & 25 & $89.3 \%$ \\
\hline & paraquat + diuron & 2 & $7,1 \%$ \\
\hline & paraquat + diuron + lambdacyalothrin & 1 & $3,6 \%$ \\
\hline & TOTAL & 28 & $100 \%$ \\
\hline Causas de & Acidental & 3 & $1^{\circ}, 7 \%$ \\
\hline \multirow[t]{4}{*}{ intoxicação } & Profissional & 10 & $35,7 \%$ \\
\hline & Suicídio & 15 & $53,6 \%$ \\
\hline & Agricultor & 17 & $60,7 \%$ \\
\hline & Ignorada & 4 & $14,3 \%$ \\
\hline Ocupação dos & Menor & 2 & $7,1 \%$ \\
\hline \multirow[t]{5}{*}{ intoxicados } & Aposentado & 1 & $3,6 \%$ \\
\hline & Bóia fria & 1 & $3,6 \%$ \\
\hline & Do lar & 2 & $7,1 \%$ \\
\hline & Outra & 1 & $3,6 \%$ \\
\hline & Cura & 13 & $46,4 \%$ \\
\hline Evolução dos & lgnorado & 3 & $10,7 \%$ \\
\hline \multirow[t]{2}{*}{ casos } & Óbito ( todos por suicídio ) & 11 & $39,3 \%$ \\
\hline & Em andamento & 1 & $3,6 \%$ \\
\hline
\end{tabular}

FONTE: PARANÁ. Secretaria de Estado da Saúde. SESA - PR Centro de Saúde Ambiental.

A tabela quatro apresenta osmunic ípios onde ocorreram as intoxicaçõese o número de casos em cada localidade, nos anos de 1998 e 1999. 
TABELA 4: MUNIC ÍPIOS EM QUE OCORRERAM OS CASOS NOSANOS DE 1998 e 1999.

\begin{tabular}{|c|c|c|c|}
\hline \multicolumn{2}{|c|}{1998} & \multicolumn{2}{|c|}{1999} \\
\hline MUNIC ÍPIO & NÚMERO DE CASOS & MUNIC ÍPIO & NÚMERO DE CASOS \\
\hline Antônio Olinto & 1 & Cel. Domingos Soares & 1 \\
\hline Campina do Simão & 2 & Floresta & 1 \\
\hline Candoi & 1 & Guaíra & 2 \\
\hline Cel. Domingos Soares & 1 & Irati & 1 \\
\hline Cel.Vivida & 2 & Lapa & 1 \\
\hline Cruz Machado & 2 & Londrina & 1 \\
\hline Guaraniaçu & 1 & Nova Tebas & 1 \\
\hline Honónio Serpa & 1 & Outro estado & 1 \\
\hline Irati & 1 & Pato Branco & 1 \\
\hline Lindoeste & 1 & Porto Barreiro & 1 \\
\hline Marquinho & 1 & Prudentópolis & 1 \\
\hline Morretes & 1 & Rio Bonito do lguaçú & 1 \\
\hline Nova Laranjeiras & 1 & São J oão & 2 \\
\hline Palmital & 1 & Santa Lúcia & 1 \\
\hline Pinhão & 1 & Outro & 1 \\
\hline Rebouças & 1 & & \\
\hline São Mateus do Sul & 1 & & \\
\hline TOTAL & 20 & TOTAL & 17 \\
\hline
\end{tabular}

FONTE: PARANÁ. Secretaria de Estado da Saúde. SESA - PR Centro de Saúde Ambiental.

\section{CONCLUSÃO}

As informações obtidas na Secretaria da Agricultura e Abastecimento do Paraná demonstram que o paraquat encontra-se entre os seis herbic idas ma is utilizados no Estado atrás apenas do glifosa to e o 2,4D.

Para o levantamento das intoxicações ocomidas no Estado, utilizou-se informações do Centro de Saúde Ambiental para os anos de 1998,1999 e 2000 (ver tabelas 1, 2 e 3).

No ano de 1998 foram constatados 20 casos de intoxicações sendo: 55\% por exposição profissional ao produto; $30 \%$ porsuic ídio e $15 \%$ exposição acidental, sendo que a maioria dos intoxicados eram agricultores, perfazendo um total de $85 \%$.

No ano de 1999 foram constatados 17 casos de intoxicações sendo: 41,1\% por exposição profissional a o produto; 35,3\% porsuic íd io, 11,88\% exposição acidentale causas indeteminada 5,9\%, a maionia dos intoxic ados eram agricultores, perfazendo um totalde $70,60 \%$.

No ano de 2000 foram constatados 28 casos de intoxicações sendo: 35,7\% por exposição profissional ao produto; $56,6 \%$ por suicídio e 10,70\% exposição acidental, a maioria dos intoxicados eram agricultores, perfazendo um total de $60,70 \%$.

Os dados analisados indicam que no Estado do Paraná a grande maioria das intoxic ações porpara quat oc orre em a gricultorese qua se sempre porexposição profissional. O suicídio é uma importante causa de intoxicação ficando em segundo lugar nos dois primeiros anos e em primeiro lugar no ano de 2000. 
Outra conclusão importante ao ana lisaros dados da evolução dos casosé a de que os óbitos ocomidos durante os três anos foram todos por suicídio o que faz deste uma importante causa de mortalidade, suscitando maior controle e precauções.

Tendo em vista os dados obtidos, medidas emergenciais devem ser tomadas no sentido educativo, para evitar, prevenir e esclarecer sobre os riscos do uso incorreto dos herbicidas.

Agradecimentos: à Secretaria de Estado da Agricultura e Abastecimento (SEAB-PR) e ao Centro de Saúde Ambiental (Secretaria de Estado da Saúde) pelasinformações fomecidas.

5 REFERÊNCIAS

1. ALMEIDA, F. S; RODRIGUES, B. N. Guia de herbicidas: contribuição para o uso adequado em plantio direto e convencional. Londrina : IAPAR 1985.

2. CAVAШ, B. M. PARAQUAT. Curitiba, 2001. Monografia (Bolsa Permanência na Disciplina de Toxicologia) Departamento de Medicina Forense e Psiquiatria. Universidade Federal do Paraná.

3. CASAREIT and DOUL'S. Toxicology: the basic science of poisons. New York: Mac Graw Hill, 1996.

4. EUENHORN, M. Ellenhorn's medical toxic ology: diagnosisand treatment of human poisoning. $2^{\text {nd }}$ ed. Baltimore: Willians \& Wilkins, 1997.

5. LARINI, L Toxicologia. São Paulo: Manole, 1997.

6. MIDIO, A. F; MARTINS, D. I. Herbicidas em a limentos. São Paulo: Livraria Varela, 1997.

7. PARANÁ. Secretaria de Estado da Agricultura e Abastecimento. Lista de agrotóxic os aptos para uso e comércio no Paraná. Disponível em : <http://www.prgov.br/seab/> Acesso em : 27 jul. 2001.

8. SINDICATO NACIONAL DA INDÚSTRIA DE DEFENSIVOS AGRÍCOLAS (SINDAG). Vendas mensais de defensivos agnícolas. Disponível em : <http://www.sindag.com.br/html/stats_agosto.html>. Acesso em : 07 nov. 2000. 